\title{
Das Mikrobiom als Therapieziel bei funktionellen und chronisch entzündlichen Magen-Darm-Erkrankungen
}

Autoren: Jost Langhorst, Anna K. Koch

\section{ZUSAMMENFASSUNG}

Das Mikrobiom spielt eine wichtige Rolle im Krankheitsgeschehen funktioneller sowie chronisch entzündlicher Darmerkrankungen (CED). Im Zuge der gesteigerten Aufmerksamkeit für das Mikrobiom wurde nach der Etablierung der Probiotikatherapie in den S3-Leitlinien für Colitis ulcerosa und das Reizdarmsyndrom auch die Therapieform des fäkalen Mikrobiotatransfers wiederentdeckt. Die standardisierte Anwendung im klinischen Alltag für die Indikationen CED und Reizdarmsyndrom ist jedoch noch nicht in Aussicht. Weitere Ansätze mit potenziell positiven Effekten auf das Mikrobiom bieten darüber hinaus Phytotherapeutika. Bei Patienten, die an funktionellen oder chronisch entzündlichen Darmbeschwerden leiden, können diese das Behandlungsspektrum wirkungsvoll ergänzen und erweitern.

\section{Schlüsselwörter}

Mikrobiom, CED, Fäkaler Mikrobiota-Transfer, Probiotika, Phytotherapie.

\section{ABSTRACT}

The microbiome plays an important role in the disease process of functional as well as chronic inflammatory bowel diseases. As a result of the increased attention paid to the microbiome, the therapy of the fecal microbiota transfer has been rediscovered after the establishment of the probiotics therapy in the $\mathrm{S3}$-guidelines for ulcerative colitis and irritable bowel syndrome. However, there is still no prospect for a standardized application in daily clinical practice for the indications CID and irritable bowel syndrome. In addition, phytotherapeutic agents offer further approaches with potentially positive effects on the microbiome. They can effectively complement and expand the treatment spectrum in patients suffering from functional or chronic inflammatory intestinal complaints.

Keywords

Microbiome, CID, fecal microbiota transfer, probiotics, phytotherapy.

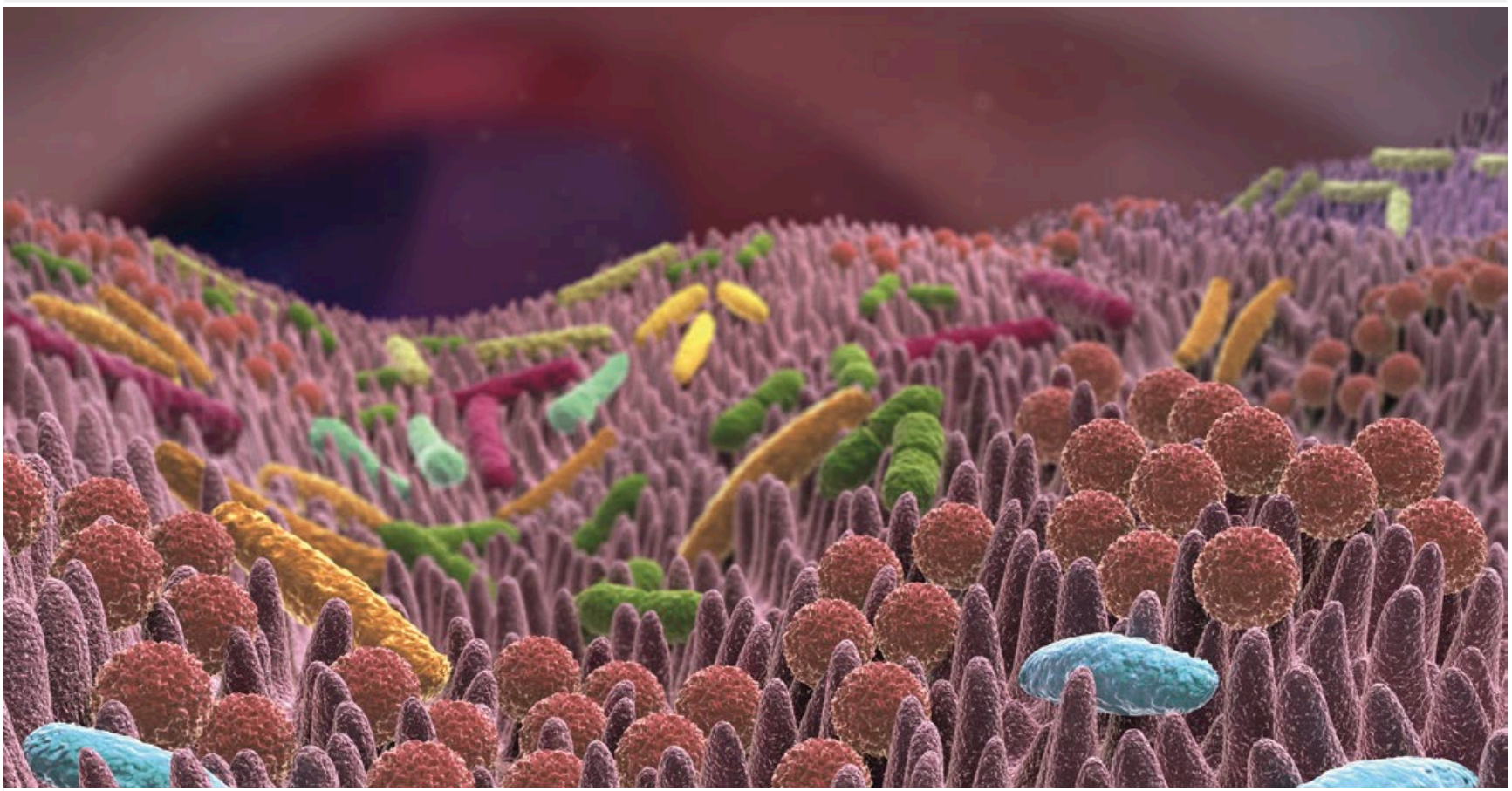

-Abb. 1 Chronisch entzündliche Magen-Darm-Erkrankungen sind weit verbreitet. Für ihre Behandlung spielt das Mikrobiom eine immer wichtigere Rolle. @ Alex/Fotolia

Funktionelle und chronisch entzündliche Magen-Darm-Erkrankungen (CED) wie das Reizdarmsyndrom (RDS), Colitis ulcerosa (CU) und Morbus Crohn (MC) sind häufige Krankheitsbilder in Industrienationen wie Deutschland [1-3].
Von CU und MC sind in Deutschland etwa 400000 Menschen betroffen. Die Kriterien für das Reizdarmsyndrom erfüllen bis zu $10 \%$ der Bevölkerung. 


\section{Symptome und Risikofaktoren}

\section{Reizdarmsyndrom}

Typische Symptome des RDS sind Bauchschmerzen, Blähungen und Verstopfung oder Durchfälle. Für die klinische Diagnostik müssen laut deutscher S3-RDS-Leitlinie der AWMF folgende 3 Kriterien erfüllt sein: 1 . das Leiden ist chronisch, dauert bereits länger als 3 Monate an, ist von Arzt und Patient auf den Darm bezogen und kann mit Stuhlgangveränderungen einhergehen, 2. die Beschwerden führen zur Hilfesuche und reduzierter Lebensqualität und 3. es gibt keine nachweisbare andere Ursache. Das RDS kann für die Betroffenen sehr belastend sein, hat aber keinen Einfluss auf die Lebenserwartung und ist nicht mit der Entwicklung anderer gastrointestinaler oder anderer schwerwiegender Erkrankungen korreliert. Bei konventioneller Diagnostik können keine organischen Ursachen für das RDS gefunden werden.

Risikofaktoren, an einem RDS zu leiden sind [2]:

- genetische Veranlagung

- externe Krankheitserreger

- Veränderung von Mikrobiom und Darmbarriere

- psychische und soziale Faktoren

- Umwelteinflüsse

- Störung des Immunsystems

- Störung der viszeralen Sensibilität

- Störungen des autonomen / zentralen Nervensystems

- Störung des enterischen Nervensystems

- Motilitätsstörung

\section{Chronisch entzündliche Magen-Darm-Erkrankungen}

CED können in sehr unterschiedlichen Schweregraden auftreten und verlaufen meist schubförmig. $\mathrm{CU}$ ist eine wiederkehrende, entzündliche und mit Ulzerationen einhergehende Erkrankung des Kolons. Typische Symptome sind blutige Durchfälle und abdominelle Schmerzen. MC ist definiert als chronisch entzündliche Erkrankung des Verdauungstrakts und kann vom Mund bis zum Anus auftreten. Typische Symptome des MC sind krampfartige Schmerzen, Durchfall und Appetitlosigkeit. Weiterhin können Hautveränderungen, Gelenkschwellungen und Augenentzündungen auftreten. Sowohl CU als auch MC können mit teils schwerwiegenden Komplikationen einhergehen. Bei CU sind dies v. a. Anämie und die Entwicklung eines kolorektalen Karzinoms. Sehr selten aber potenziell lebensbedrohlich ist die Entwicklung eines toxischen Megakolons (pathologische akute Ausdehnung des Dickdarms). Bei MC sind mögliche Komplikationen v. a. Stenosen, Abszesse und Fisteln.

Verschiedene Risikofaktoren, an einer CED zu erkranken, sind beschrieben, die Ätiologie dieser Erkrankungen ist allerdings noch nicht abschließend geklärt. Wahrscheinlich ist ein multifaktorielles Geschehen als Ursache. Risikofaktoren sind demnach zu suchen in den Bereichen [1, 3]:

- genetische Faktoren

- Mikrobiom und Darmbarriere

- Immunsystem

- Psyche und soziale Faktoren

- Umweltfaktoren

\section{Probiotika als Therapieoption}

Aufgrund der multifaktoriellen Ätiologie sowohl beim RDS als auch bei CED ist es naheliegend, dass Patienten von einer individuell zugeschnittenen multimodalen Therapie profitieren. Im akuten Schub von CED ist die Behandlung der Primärsymptomatik mit pharmakologisch-synthetischen Präparaten unter Überwachung eines Arztes von zentraler Bedeutung. Ergänzend gibt es eine Vielzahl von Methoden aus dem Bereich der Komplementärmedizin (CAM), welche das Behandlungsspektrum wirkungsvoll ergänzen und erweitern. Der Einsatz sollte immer in Rücksprache mit dem behandelnden Arzt erfolgen.

Ein aktuell zunehmend beachteter Ansatzpunkt in der Therapie, der sowohl dem RDS als auch den CED gemein ist, ist das Mikrobiom. Verschiedene komplementäre Therapieformen haben eine Wirkung auf das Mikrobiom. Darunter die Therapie mit Probiotika, welche eine lange naturheilkundliche Tradition haben. Die S3-Leitlinie der AWMF zur Therapie der CU empfiehlt beispielsweise zur Remissionserhaltung als Therapieoption eine Behandlung mit dem Escherichia coli Stamm Nissle 1917. Bei Kindern wird hier das probiotische Präparat VSL\#3 empfohlen. Zur Primärprophylaxe der Pouchitis kann weiterhin das probiotische Kombinationspräparat VSL\#3 (verschiedene Laktobacili, Bifidobakteriae und Streptokokken) eingesetzt werden. Hinsichtlich MC existieren nur sehr kleine Studien, welche aktuell keine probiotische Empfehlung ermöglichen. Zur Behandlung des RDS hingegen sind die Empfehlungen zum Einsatz von Probiotika nicht zuletzt aufgrund der breiteren Studienlage deutlich vielfältiger. Allerdings ist keine pauschale Aussage möglich, dass Probiotika zur Therapie des RDS wirksam sind. Eine Differenzierung zwischen den verschiedenen probiotischen Spezies bzw. Stämmen für einzelne Patientengruppen ist nötig ( $\triangleright$ Tab. 1).

\section{Fäkaler Mikrobiotatransfer}

Im Zuge der gesteigerten Aufmerksamkeit wird derzeit zudem eine Therapieform wiederentdeckt und diskutiert, die seit Jahrtausenden Anwendung fand und jetzt dank moderner Technik differenzierter evaluiert werden kann: der fäkale Mikrobiotatransfer (umgangssprachlich auch „Stuhltransplantation“). Beim fäkalen Mikrobiotatransfer wird der Stuhl eines gesunden Spenders in den MagenDarm-Trakt eines erkrankten Patienten übertragen. Hierdurch erhofft man sich eine Besserung der Symptome oder gar die Heilung des Patienten. Dieses Therapieverfahren ist 
- Tab. 1 Evidenzgrade: Empfehlungen zur Therapie des Reizdarmsyndroms mit Probiotika [2].

\begin{tabular}{|l|l|l|l|}
\hline Probiotika-Stamm & $\begin{array}{l}\text { RDS } \\
\text { Schmerz/Bläh-Typ }\end{array}$ & $\begin{array}{l}\text { RDS } \\
\text { Schmerztyp }\end{array}$ & $\begin{array}{l}\text { RDS } \\
\text { Obstipationstyp }\end{array}$ \\
\hline Bifidobacterium infantis 35624 & B & & \\
\hline Bifidobacterium animali ssp. Lactis DN-173010 & B & C \\
\hline Lactobacillus casei Shirota & B & B \\
\hline Lactobacillus plantarum & C & B & \\
\hline Lactobacillus rhamnosus GC & & & C \\
\hline E. coli Nissle 1917 & & C & \\
\hline Kombinationspräparate & & \\
\hline 1 Nur an Kindern gezeigt. & & \\
\hline
\end{tabular}

bei Clostridium-difficile-Infektionen in die aktuelle Leitlinie der AWMF als Therapieoption bei Antibiotika-resistenten Verläufen aufgenommen worden. Bei RDS und CED ist die Therapie in Deutschland allerdings (noch) nicht zugelassen und birgt viele potenzielle Komplikationen und Risiken. Neben den gewünschten heilungsfördernden Bestandteilen der Mikrobiota (z. B. Bakterien oder anti-inflammatorische Proteine) können auch unerwünschte Stoffe oder prädisponierende Faktoren für Krankheiten (Bakterien, Parasiten, Viren, proentzündliche Proteine) übertragen werden. Da an den Spender strenge Kriterien gestellt werden, ist die Gefahr einer solchen ungewollten Krankheitsübertragung zwar verringert, ob und wie sie ganz ausgeschaltet werden kann ist allerdings noch nicht hinreichend geklärt. Ein weiteres Problem ist die „Kompatibilität“ von Spender und Empfänger. Es gibt erste Hinweise, dass neben der Auswahl qualitativ hochwertiger Spender auch die Passung zwischen Spender und Empfänger eine Rolle spielt. Die Mikrobiotaspende eines gesunden Probanden führte beispielsweise bei verschiedenen Patienten zu unterschiedlichen Reaktionen, was die Vermutung nahelegt, dass die Herstellung eines universell einsetzbaren Mikrobiotapräparats wohl utopisch ist [4].

Ein Ansatz, um diese Probleme differenzierter anzugehen, ist die Herstellung von speziellen Bakterien- und Probiotikacocktails. So könnte ein individuell zusammengestellter Cocktail beispielsweise in Pillenform verabreicht werden, um der Problematik der Risiken und Passgenauigkeit zwischen Spender und Empfänger besser gerecht zu werden. Die Forschung hierzu steckt aber noch in den Kinderschuhen. Somit gibt es viele Fragen, die wissenschaftlich beantwortet werden müssen, bevor eine standardisierte Anwendung des Therapieverfahrens denkbar ist:

- Auswahl des Spenders

- Passung Spender - Empfänger

- Vorbereitung des „Transplantats“

- Vorbereitung des Empfängers

- Form der Verabreichung des Mikrobiotatransfers

- Anzahl der Wiederholungen der Therapie

- Langzeitfolgen und Nebenwirkungen

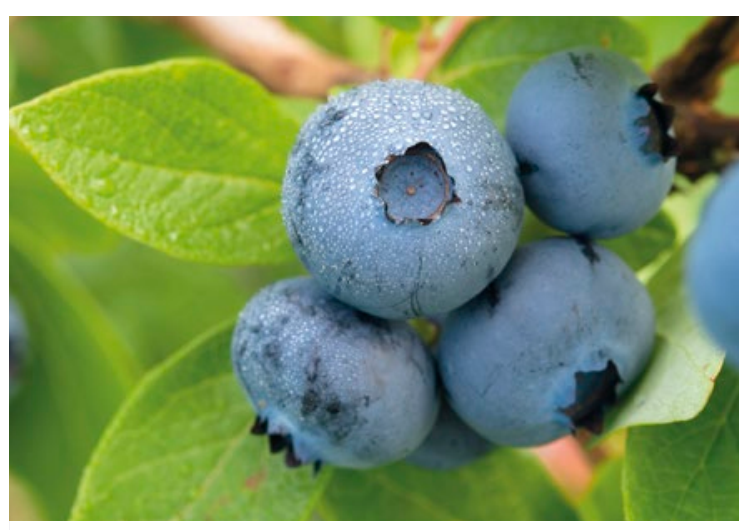

- Abb. 2 Erste Ergebnisse klinischer Studien zeigen positive Ergebnisse für die Behandlung von CED und RDS mit Phytotherapeutika wie Kamille, Heidelbeeren oder Pfefferminze. (c) Andris T/Fotolia

Randomisiert kontrollierte Studien (RCT) zur Anwendung des fäkalen Mikrobiotatransfers sind rar. Zur CU liegen bisher drei RCT [5-7] vor, von denen zwei ein signifikant positives Ergebnis mit allerdings geringer Effektstärke zeigen. Für MC und das RDS liegen bisher nur In-vitro- sowie Fallstudien vor, RCT sind allerdings in Vorbereitung. Aktuelle Reviews kommen anhand der Betrachtung dieser Studien zu dem Schluss, dass die Anwendung fäkaler Mikrobiotatransfers bei Magen-Darm-Erkrankungen Potenzial hat, allerdings weitere qualitativ hochwertiger RCT erforderlich sind, um standardisierte Leitlinien zur Anwendung zu entwickeln [8-10]. Der fäkale Mikrobiotatransfer bei CED und RDS sollte deshalb in Deutschland aktuell nur in randomisiert-kontrollierten Studien durchgeführt werden, um einen möglichst großen Wissenszuwachs in Hinblick auf Sicherheit, Studiendurchführung und Outcome zu gewährleisten.

\section{Einsatz von Phytotherapeutika}

Daneben liegen weitere Therapieoptionen vor, welche ihren Therapieeffekt potenziell ebenfalls über Wechselwirkung mit dem Mikrobiom entwickeln. Speziell ist hier 
neben der Ernährung v. a. die Phytotherapie von Bedeutung. Phytotherapeutika stellen eine vielversprechende Therapieoption bei Magen-Darm-Erkrankungen dar [11]. Zudem gibt es erste Hinweise darauf, dass sich phytotherapeutische Präparate in ihrer Wirkung auf das Mikrobiom von konventionellen Medikamenten [12,13] unterscheiden. Die Therapie mit Myrrhe, Kamillenblütenextrakt und Kaffeekohle bspw. scheint bei Patienten mit Colitis ulcerosa während eines Schubes andere Effekte auf die für das Krankheitsbild relevanten kurzkettigen Fettsäuren im Stuhl auszuüben, als die Therapie mit Mesalazin. Patienten, die mit Mesalazin behandelt wurden, wiesen im Vergleich zur Remission im akuten Schub signifikant weniger kurzkettige Fettsäuren im Stuhl auf [12]. In der Patientengruppe, die mit dem pflanzlichen Kombinationspräparat behandelt wurde, ließ sich dieser signifikante Abfall der kurzkettigen Fettsäuren bemerkenswerterweise nicht nachweisen. Kurzkettige Fettsäuren sind in verschiedene gastrointestinale Prozesse involviert - u. a. spielen sie für das Immunsystem eine wichtige Rolle, sind für die Mucinbildung von Bedeutung, unterstützen die Nährstoffabsorption und die Nutrition der Darmschleimhaut und haben krankheitsprotektive Eigenschaften [14-16]. Daher sind diese Ergebnisse hoch interessant und eröffnen im besten Falle den Zugang zu einem neuen Verständnis der Wirkmechanismen. Inwieweit Phytotherapeutika weitere positive Effekte auf das Mikrobiom haben, bleibt zu klären. Der überwiegende Teil pflanzlicher Präparate ist bisher auf mögliche Effekte auf das Mikrobiom erkrankter Patienten nicht klinisch evaluiert. Es gibt aber eine Reihe von Phytotherapeutika, für die ersten positiven Ergebnisse in klinischen Studien vorliegen und die im Rahmen der aktuellen S3-Leitlinine-Aktualisierungen für CED und das RDS diskutiert werden.

\section{Phytotherapeutika bei CED}

Myrrhe, Kamille, Kaffeekohle (Myrrhinil intest ${ }^{\circledR}$ ) [17]

- Myrrhe wirkt entzündungshemmend. Bei CED findet es als Kombinationspräparat Anwendung. Unverdünnt kann es zur Behandlung von Aphten eingesetzt werden.

- Myrrhe-Trockenextrakt aus dem Harz der Rinde: u. a. Commiphora-Säure

- gute Verträglichkeit, selten allergische Reaktionen

- Kamille-Trockenextrakt, selten allergische Reaktion

- Kaffeekohle besteht aus den gemahlenen, bis zur Schwarzbräunung und Verkohlung der äußeren Samenpartien gerösteten grünen, getrockneten Früchten von Coffea arabica

- Anwendung in Kombination: Myrrhe-Harz (100 mg), Trockenextrakt aus Kamilleblüten (70 mg) und Kaffeekohle (50 mg); Dosierung: 3-mal tgl. 4 Tbl.; Myrrhetinktur zur Therapie oraler Aphten oder einer Stomatitis

- Zulassung in Deutschland als traditionelles Arzneimittel

- Erstes RCT für Remissionserhaltung bei Colitis ulcerosa

Gelbwurz (Curcumae longae rhizoma) [18-20]

- Trockenextrakt aus dem Curcuma-Wurzelstock

- Wirkstoffe: Curcuminoide (Turmeric) und das ätherische Öl

- gute Verträglichkeit, selten allergische Reaktionen

- mittlere Tagesdosis: 1,5-3g Drogenpulver; Tinktur; 2×1 Kaps. tgl. (81 mg standardisiertes Extrakt) als Fertigpräparat

- jeweils eine RCT für Remissionserhaltung und für Remissionsinduktion bei Colitis ulcerosa

- in Deutschland nur als Nahrungsergänzungsmittel verfügbar 
Flohsamen (Psyllii semen) [21]

- gemahlene Flohsamenschalen

- Quellmittel bzw. Gelbildner über Wasserbindung

- darf nicht eingesetzt werden bei bekannten Stenosen

- mögliche Bindung und damit Wirkungsherabsetzung gleichzeitig eingenommener Medikamente, deshalb hier mindestens 1 Stunde zeitlicher Abstand bei der Einnahme

- Dosierung: 1-3-mal tgl. ein Beutel à $5 \mathrm{~g}$

- eine RCT für Remissionserhaltung bei Colitis ulcerosa

- Zulassung als Arzneimittel: Erstattungsfähig bei M. Crohn (hierzu jedoch keine Studie)

Heidelbeeren (Vaccinium myrtillus) [22]

- Wirkstoff: u. a. reich an Anthocyanen

- eignen sich besonders für den Einsatz bei akuten Schüben mit Diarrhöen, z. B. in Form von kalt gepresstem Muttersaft

- Dosierung: 1-3-mal tgl. 100 ml als Heidelbeermuttersaft oder getrocknete Früchte

- Eine Fallserie zeigt erste Hinweise für eine positive Wirkung von Heidelbeeren bei aktiver Colitis ulcerosa; qualitativ hochwertige RCTs sind allerdings nötig, um dies zu bestätigen.

Wermut (Artemisia absinthum) [23, 24]

- v. a. bei Morbus Crohn

- wirkt über Bitterstoffe u. a. Artemisinin

- seltene Nebenwirkungen sind gastrointestinale Beschwerden oder in Einzelfällen die Senkung der Krampfschwelle

- ein in Studien untersuchtes Präparat ist in Deutschland nicht zugelassen

- in Leitlinien bisher keine Empfehlung; Verwendung als Tee (sehr bitter!)

Weihrauch (Boswellia serrata) [25-28]

- wirkt potenziell entzündungshemmend

- Potenzial zur unterstützenden Therapie bei Morbus Crohn v. a. bei gleichzeitig auftretenden Gelenkbeschwerden; auch für Colitis ulcerosa erste klinische Daten

- Trockenextrakt aus dem Harz der Rinde

- Hauptwirkstoff: Boswelliasäure

- gute Verträglichkeit, selten allergische Reaktionen

- Dosierung: 3-mal tgl. 2 á 400 mg

- Präparate in Deutschland nur als Nahrungsergänzungsmittel verfügbar

Indische Echinacea (Andrographis paniculata) [29, 30]

- bitter schmeckende einjährige Pflanze, verbreitet in großen Teilen Asiens

- Wirkstoffe: Diterpene und Lactone

- Nebenwirkungen: Beschrieben wurden z. B. Kopfschmerzen, Müdigkeit, allergische Reaktionen, Lymphknotenschwellungen, Schmerzen in den
Lymphknoten, Übelkeit, Durchfall und Geschmacksveränderungen

- Präparate in Deutschland nicht verfügbar

Weizengrassaft (Triticum aestivum) [31]

- Wirkstoffe: Vitamine und Mineralien

- Wirkmechanismus unklar

\section{Phytotherapeutika bei RDS}

Pfefferminze [32]

- Es gibt zahlreiche Arten und Darreichungsformen von Pfefferminze. Der Hauptwirkstoff ist Menthol.

- Pfefferminztee: wird bei krampfartigen Beschwerden des Magen-Darm-Trakts sowie Übelkeit, Brechreiz und Erbrechen eingesetzt

- ätherisches Pfefferminzöl: wird v. a. bei Kopfschmerzen auf die Schläfen gerieben

- ätherisches Pfefferminzöl in magensaftresistenten Kapseln: kann zur Behandlung des Reizdarmsyndroms kurzfristig effektiv angewendet werden

- Nebenwirkungen: bei oraler Einnahme kann Sodbrennen auftreten, selten allergische Reaktionen

Kümmelöl-Leibauflagen [33]

- Warme Kümmelöl-Leibauflagen als externale phytotherapeutische Anwendung nach Kneipp sind eine Selbsthilfestrategie, deren Wirksamkeit und Sicherheit zur Behandlung des Reizdarmsyndroms in einer ersten RCT gezeigt werden konnte.

- Anwendung: Ein Geschirrtuch in heißem Wasser tränken und auswringen. 1 TL eines Ölgemischs (Kümmel (2-10\%) und Olivenöl; erhältlich in der Apotheke) auf den Bauch auftragen und in kreisenden Bewegungen einreiben. Hierauf eine Wärmeflasche legen und 30 min hinlegen. Falls Unverträglichkeiten wie Juckreiz auftreten, das Öl mit warmem Wasser abwischen und von weiteren Anwendungen absehen.

Kümmel- und Pfefferminzöl

- auch als Kombinationspräparat (Carmenthin ${ }^{\circledR}$ ) erhältlich, das bei der Behandlung des RDS vielversprechenden Therapieoptionen zeigt

- RCT wird aktuell durchgeführt

- Dosierung: 1-0-1

- gute Verträglichkeit, selten allergische Reaktionen

STW-5 [34]

- STW-5 (Iberogast ${ }^{\circledR}$-bestehend aus Iberis Amara, Angelikawurzel, Kamillenblüten, Kümmelfrüchten, Mariendistelfrüchten, Melissenblättern, Pfefferminzblättern, Schöllkraut und Süßholzwurzel) sowie das Folgepräparat (STW-5-II; bestehend aus Iberis Amara, Kamillenblüten, Kümmelfrüchten, Melissenblättern, Pfefferminzblättern und Süßholzwurzel) können die generelle Reizdarmsymptomatik sowie abdominelle Schmerzen lindern. 
- eine positive RCT liegt vor

- Dosierung: bis zu 3-mal tgl. 20 Tr.

- gute Verträglichkeit, selten allergische Reaktionen

Interessenkonflikte

Die Autoren geben an, dass kein Interessenkonflikt besteht.

Über die Autoren

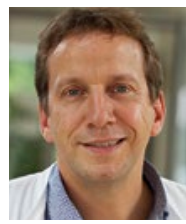

\section{Prof. Dr. Jost Langhorst}

Jost Langhorst ist Facharzt für Innere Medizin und Gastroenterologie mit den Zusatzbezeichnungen Psychotherapie und Naturheilverfahren; leitender Arzt der Abteilung Integrative Gastroenterologie der Klinik für Naturheilkunde und Integrative Medizin der Kliniken Essen-Mitte.

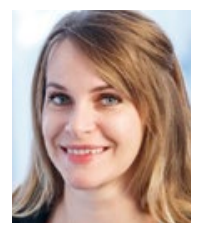

\section{Sc. Psych. Anna K. Koch}

Anna K. Koch ist Psychologin und seit Februar 2016 wissenschaftliche Mitarbeiterin der Klinik für Naturheilkunde und Integrative Medizin an den Kliniken Essen-Mitte. Neben ihrer Anstellung an den Kliniken Essen-Mitte promoviert sie am Fachbereich für Arbeitsund Organisationspsychologie der Universität Hamburg.

\section{Korrespondenzadresse}

Prof. Dr. Jost Langhorst

Klinik für Naturheilkunde und Integrative Medizin

Kliniken Essen-Mitte, Universität Duisburg-Essen

Am Deimelsberg 34a

45257 Essen

E-Mail: J.Langhorst@kliniken-essen-mitte.de

\section{Literatur}

[1] Hoffmann JC, Zeitz M. S3 guideline by the German Society of Digestive and Metabolic Diseases and the Competence Network of Chronic Inflammatory Bowel diseases on diagnosis and therapy of ulcerative colitis. An update. Med Klin 2005; 100(1): 43-50

[2] Layer P, Andresen V, Pehl C et al. Irritable bowel syndrome: German consensus guidelines on definition, pathophysiology and management. Z Gastroenterol 2011; 49(2): 237-293

[3] Preiss JC, Bokemeyer B, Buhr HJ, et al. Updated German clinical practice guideline on „Diagnosis and treatment of Crohn's disease“ 2014. Z Gastroenterol 2014; 52(12): 1431-1484

[4] Li SS, Zhu A, Benes $V$ et al. Durable coexistence of donor and recipient strains after fecal microbiota transplantation. Science 2016; 352(6285): 586-589 
[5] Moayyedi P, Surette MG, Kim PT et al. Fecal Microbiota Transplantation Induces Remission in Patients With Active Ulcerative Colitis in a Randomized Controlled Trial. Gastroenterology 2015; 149(1): 102-109.e6

[6] Paramsothy S, Kamm MA, Kaakoush NO, et al. Multidonor intensive faecal microbiota transplantation for active ulcerative colitis: a randomised placebo-controlled trial. Lancet 2017; 389(10075): 1218-1228

[7] Rossen NG, Fuentes S, van der Spek M], et al Findings From a Randomized Controlled Trial of Fecal Transplantation for Patients With Ulcerative Colitis. Gastroenterology 2015; 149(1): 110-118.e114

[8] Rossen NG, MacDonald JK, de Vries EM et al. Fecal microbiota transplantation as novel therapy in gastroenterology: A systematic review. World J Gastroenterol 2015; 21(17): 5359-5371

[9] Shi Y, Dong Y, Huang W et al. Fecal Microbiota Transplantation for Ulcerative Colitis: A Systematic Review and Meta-Analysis. PloS one 2016; 11(6):e0157259

[10] Sun D, Li W, Li S et al. Fecal Microbiota Transplantation as a Novel Therapy for Ulcerative Colitis: A Systematic Review and Meta-Analysis. Medicine 2016; 95(23):e3765

[11] Holtmann G, Talley NJ. Herbal medicines for the treatment of functional and inflammatorybowel disorders. Clin Gastroenterol Hepatol 2015; 13(3): 422-432

[12] Langhorst J, Koch AK. Distinct patterns of short chain fatty acids in patients with ulcerative colitis experiencing a flare during treatment with mesalamine or a herbal combination of myrrh, chamomile flowers and coffee charcoal. Gastroenterol 2017; 47(5), Suppl 1: 616

[13] Langhorst J, Frede A, Knott $M$ et al. Distinct kinetics in the frequency of peripheral CD4+ T cells in patients with ulcerative colitis experiencing a flare during treatment with mesalazine or with a herbal preparation of myrrh, chamomile, and coffee charcoal. PloS one 2014; 9(8):e104257

[14] Chapman MA, Grahn MF, Boyle MA et al. Butyrate oxidation is impaired in the colonic mucosa of sufferers of quiescent ulcerative colitis. Gut 1994; 35(1): 73-76

[15] Wong JM, de Souza R, Kendall CW et al. Colonic health: fermentation and short chain fatty acids. J Clin Gastroenterol 2006; 40(3): 235-243

[16] Machiels K, Joossens M, Sabino J et al. A decrease of the butyrate-producing species Roseburia hominis and Faecalibacterium prausnitzii defines dysbiosis in patients with ulcerative colitis. Gut 2014; 63(8): 1275-1283

[17] Langhorst J, Varnhagen I, Schneider SB et al. Randomised clinical trial: a herbal preparation of myrrh, chamomile and coffee charcoal compared with mesalazine in maintaining remission in ulcerative colitis--a double-blind, double-dummy study. Aliment Pharmacol Ther 2013; 38(5): 490-500

[18] Hanai H, lida T, Takeuchi K et al. Curcumin Maintenance Therapy for Ulcerative Colitis: Randomized, Multicenter, Double-Blind, Placebo-Controlled Trial. Clin Gastroenterol Hepatol. 2006; 4(12): 1502-1506

[19] Lang A, Salomon N, Wu JCY et al. Curcumin in Combination With Mesalamine Induces Remission in Patients With Mildto-Moderate Ulcerative Colitis in a Randomized Controlled Trial. Clin Gastroenterol Hepatol 2015; 13(8):1444-1449. e1441

[20] Singla V, Pratap Mouli V, Garg SK et al. Induction with NCB02 (curcumin) enema for mild-to-moderate distal ulcerative colitis - a randomized, placebo-controlled, pilot study. J Crohns Colitis 2014; 8(3): 208-214
[21] Fernandez-Banares F, Hinojosa J, Sanchez-Lombrana JL et al. Randomized clinical trial of Plantago ovata seeds (dietary fiber) as compared with mesalamine in maintaining remission in ulcerative colitis. Spanish Group for the Study of Crohn's Disease and Ulcerative Colitis (GETECCU). Am J Gastroenterol 1999; 94(2): 427-433

[22] Biedermann L, Mwinyi J, Scharl M et al. Bilberry ingestion improves disease activity in mild to moderate ulcerative colitis - an open pilot study. J Crohns Colitis 2013; 7(4): 271-279

[23] Krebs S, Omer TN, Omer B. Wormwood (Artemisia absinthium) suppresses tumour necrosis factor alpha and accelerates healing in patients with Crohn's disease - A controlled clinical trial. Phytomedicine 2010; 17(5): 305-309

[24] Omer B, Krebs S, Omer $\mathrm{H}$ et al. Steroid-sparing effect of wormwood (Artemisia absinthium) in Crohn's disease: A double-blind placebo-controlled study. Phytomedicine 2007; 14(2-3): 87-95

[25] Gupta I, Parihar A, Malhotra P et al. Effects of gum resin of Boswellia serrata in patients with chronic colitis. Planta medica 2001; 67(5): 391-395

[26] Gupta I, Parihar A, Malhotra P et al. Effects of Boswellia serrata gum resin in patients with ulcerative colitis. Europ J Med Res 1997; 2(1): 37-43

[27] Gerhardt H, Seifert F, Buvari P et al. Therapy of active Crohn disease with Boswellia serrata extract H 15. Z Gastroenterol 2001; 39(1): 11-17

[28] Holtmeier W, Zeuzem S, Preiss ] et al. Randomized, placebo-controlled, double-blind trial of Boswellia serrata in maintaining remission of Crohn's disease: good safety profile but lack of efficacy. Inflamm Bow Dis. 2011; 17(2): 573-582

[29] Sandborn W], Targan SR, Byers VS et al. Andrographis paniculata extract (HMPL-004) for active ulcerative colitis. Am J Gastroenterol 2013; 108(1): 90-98

[30] Tang T, Targan SR, Li ZS et al. Randomised clinical trial: herbal extract HMPL-004 in active ulcerative colitis - a double-blind comparison with sustained release mesalazine. Aliment Pharmacol Ther 2011; 33(2): 194-202

[31] Ben-Arye E, Goldin E, Wengrower D et al. Wheat grass juice in the treatment of active distal ulcerative colitis: a randomized double-blind placebo-controlled trial. Scand J Gastroenterol 2002; 37(4): 444-449

[32] Khanna R, MacDonald JK, Levesque BG. Peppermint oil for the treatment of irritable bowel syndrome: a systematic review and meta-analysis. J Clin Gastroenterol 2014; 48(6): 505-512

[33] Lauche R, Janzen A, Ludtke R et al. Efficacy of Caraway Oil Poultices in Treating Irritable Bowel Syndrome - A Randomized Controlled Cross-Over Trial. Digestion 2015; 92(1): 22-31

[34] Madisch A, Holtmann G, Plein K et al. Treatment of irritable bowel syndrome with herbal preparations: results of a double-blind, randomized, placebo-controlled, multi-centre trial. Aliment Pharmacol Ther 2004; 19(3): 271-279

\section{Bibliografie}

DOI https://doi.org/10.1055/s-0043-109501

EHK 2017; 66: 140-146

(c) MVS Medizinverlage Stuttgart GmbH \& Co. KG ISSN 0014-0082 